\title{
Design of Novel Reconfigurable Reflectarrays with Single-bit Phase Resolution for Ku-Band Satellite Antenna Applications
}

\author{
Ming-Tao Zhang, Member, IEEE, Steven Gao, Member, IEEE, Yong-Chang Jiao, Senior Member, \\ IEEE, Ji-Xiang Wan, Bu-Ning Tian, Chun-Bang Wu, and Andrew-John Farrall, Student Member, IEEE
}

\begin{abstract}
Reconfigurable reflectarray antennas operating in Ku-band are presented in this paper. First, a novel multilayer unit-cell based on polarization turning concept is proposed to achieve the single-bit phase shift required for reconfigurable reflectarray applications. The principle of the unit-cell is discussed using the current model and the space match condition, along with simulations to corroborate the design and performance criteria. Then, an offset-fed configuration is developed to verify performance of the unit-cell in antenna application, and its polarization transformation property is elaborated. Finally, an offset-fed reflectarray with $10 \times 10$ elements is developed and fabricated. The dual-polarized antenna utilizes the control code matrices to accomplish a wide angle beam-scanning. A full wave analysis is applied to the reflectarray, and detailed results are presented and discussed. This electronically steerable reflectarray antenna has significant potential for satellite applications, due to its wide operating band, simple control and beam-scanning capability.
\end{abstract}

Index Terms-Reflectarrays, reconfigurable antennas, single-bit, phase shift, polarization twist, beam-scanning.

\section{INTRODUCTION}

$\mathrm{R}$ ECONFIGURABLE reflectarray antennas (RRAs) are an emerging technology that has recently shown promise for advanced satellite communications. For space applications, reconfigurability of antennas is critical for flexible payload, due to the potential changes of requirements and multifunctional operation modes during the satellites lifetime. Space antennas with beam-scanning or beam-switching capabilities are anticipated for future communications systems. RRAs offer the beam-scanning function with high gain and high resolution, making them an ideal choice for space applications. Compared with active phased array antennas, RRAs show potential for reducing cost of space antennas, which will enable

This work was supported by China Scholarship Council and Xi'an Institute of Space Radio Technology.

M.-T. Zhang is with the Xi'an Institute of Space Radio Technology, Xi'an 710100, P. R. China and also with the School of Engineering and Digital Arts, University of Kent, Canterbury, CT2 7NT, UK (email: mtzhang@126.com).

S. Gao and A.J. Farrall are with the School of Engineering and Digital Arts, University of Kent, Canterbury, CT2 7NT, UK (email: s.gao@kent.ac.uk).

Yong-Chang Jiao is with the National Key Laboratory of Antennas and Microwave Technology, Xidian University, Xi'an 710071, P. R. China (email: ychjiao@xidian.edu.cn).

J.X. Wan, B.-N. Tian and C.-B. Wu are with the Xi'an Institute of Space Radio Technology, Xi'an 710100, P. R. China. development of larger aperture antennas with higher resolutions. The loss increment in the beam-forming network and the high cost in active phased array antenna systems make them less favorable. In recent years, many RRAs [1] were proposed, in which PIN diodes, RF MEMS, varactors, liquid crystals (LC) and ferroelectric thin films are used [2]. RRAs are now a viable alternative choice of antenna offering not only beam-steering capabilities, but also many other flexible functions.

Typically, for a RRA, there is a trade-off between two major factors: reconfigurability and complexity. Normally a fast pattern reconfiguration is necessary for realizing flexible real-time radio systems, and for switching between different antenna operational modes. High performance requirements, such as high directivity and narrow beam, imply that the antennas need very large apertures. These properties can be achieved by large antennas with electronic beam scanning in wide angle ranges, however this increases complexity and hence the cost. Reflectarrays with single-bit phase shift for beam-scanning [3-5] have attracted attention due to their ability for balancing between the pattern reconfiguration and the beam-control simplification. Thus, elementary cells with single-bit phase resolution $\left(0^{\circ} / 180^{\circ}\right)$ are identified as a means for realizing and controlling RRAs. Based on the polarization turning concept, the elementary cells could achieve a flat phase-shift response in the operating pass band defined by the impedance match and power conversion between two orthogonal polarizations, compared with designs based on the tunable resonator approach [6].

In this paper, a novel Ku-band RRA based on a new type of polarization turning unit-cell, with single-bit phase resolution is proposed. By using the unit-cell, a linearly polarized incident wave can be transformed into the orthogonal linearly polarized reflected wave in the operating frequencies. Based on the polarization turning technique, PIN diodes in the unit-cell along with its symmetrical structure, allows switching between two states of $+5 \mathrm{~V}$ and $-5 \mathrm{~V}$ (biasing voltages), giving the ability of a 1-bit phase-resolution. Thanks to its symmetrical structure, the dual-polarized unit-cell for RRA can not only reflect the linearly polarized incident wave, but also form the circularly polarized wave. The proposed unit-cell has a number of technical advantages compared with existing unit-cells. In the offset-fed reflectarray configuration, the beam is scanning by controlling biasing voltages among the array elements, and the 
circularly polarized beam may also be formed when the horn feed is circularly polarized.

This paper is organized as follows. In Section II, the novel unit-cell and its scattering characteristics are presented. In Section III, the offset-fed RRA with the proposed unit-cell is presented. The offset-fed RRA is analyzed in Section IV, and the simulated results are evaluated. Measurements are also presented to verify the design. We finally conclude our paper in Section V.

\section{THE REFLECTARRAY UNIT-CELL}

The conventional RRA design based on the tunable approach [6] contributes a frequency dependent phase shift that will degrade antenna performance for applications designed to cover the complete $\mathrm{Ku} \mathrm{T} x / \mathrm{Rx}$ band. Therefore, the polarization turning concept introduced in this paper is preferred because it provides a flat phase-shift response that is independent of frequency in the operating band defined by the impedance match and power conversion. In two states corresponding to positive and negative voltages, polarization of the reflected electric fields is twisted by $\pm 90^{\circ}$ angle with respect to the linear polarization of the incident field, however the state is distinguished by the polarization-twist direction. Because of its symmetrical structure, the electric field of the reflected waves from this unit-cell in both states after the polarization turning is in the same line, but of opposite direction. That is to say, the corresponding phase difference between these two states is $180^{\circ}$, thus the 1-bit phase shift can be realized based on the polarization turning concept for RRAs. The different direction of the polarization twist is controlled by PIN diodes, and the symmetrical structure endows the unit-cell with 1-bit phase resolution for RRAs.

In the RRA unit-cell design, the multilayer PCB structures with an air gap layer, resonant patches and the coaxial DC voltage circuit are built. Configuration diagrams are shown in Fig. 1. The multi-layer structure consists of Rogers Duroid 5880 substrates of different thicknesses with relative permittivity $\varepsilon_{\mathrm{r}}=2.2$ and low loss tangent. Defined by their functions, the unit-cell mainly consists of 4 parts, the impedance-match layer (0.508-mm thick), the polarization-turning layer (0.508-mm thick), the coupling layer (0.787-mm thick), and the RF-DC isolation circuitry. Four PIN diodes are placed on 4 microstrip lines in the polarization-turning layer to obtain a Single Pole Double Throw (SPDT) configuration, which supplies two states for reflecting the linearly polarized incident wave. Different orientations of 2 pairs (each with 2 PIN diodes) fixed on the radial line can turn the linear polarization of the incident wave into its orthogonal linear polarization.

In the performance assessment, equivalent circuits for the applied PIN diodes including package effects, such as series inductance, total reverse bias capacitance at $-5 \mathrm{~V}$ and forward series resistance at $+5 \mathrm{~V}$, provided by the manufacturer, are considered and modeled. PIN diode losses are also taken into account. Special settings of PIN diodes give the unit-cell two different RF parameters at corresponding $+5 \mathrm{~V}$ or $-5 \mathrm{~V}$ bias voltage. Four PIN diodes in Fig. 1 (b) are defined by numbers in
$\{1,2,3,4\}$. If $+5 \mathrm{~V}$ biasing voltage is exerted at the control point, corresponding to state "1", the diode array will manifest state \{"off", "on", "off", "on"\} in the diode-switch array. While $-5 \mathrm{~V}$ biasing voltage is exerted at the control point defined as state " 0 ", the diode array will manifest state ‘"on", "off", "on", "off" $\}$. Two states of bias voltages, forward voltage and reverse bias voltage, are switched for each pair of PIN diodes. This enables the unit-cell to control the induced currents and the reflected electric field by applying different biasing voltages, $+5 \mathrm{~V}$ and $-5 \mathrm{~V}$.

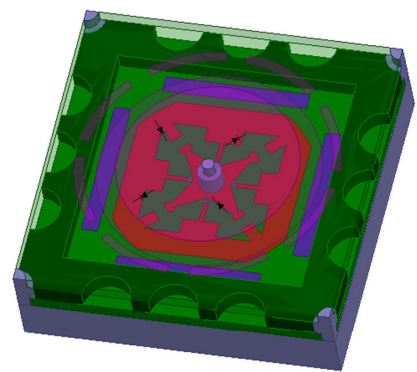

(a)

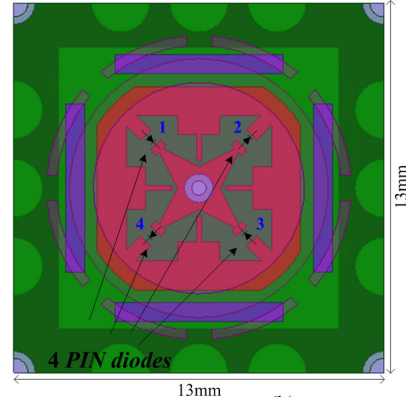

(b)

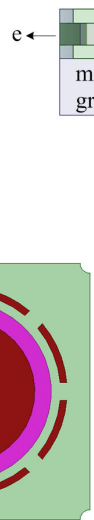

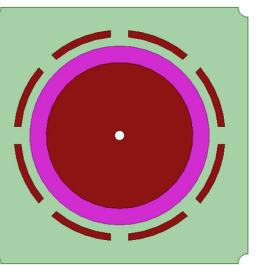

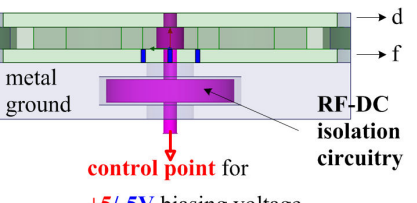

$+5 /-5 \mathrm{~V}$ biasing voltage

(c)

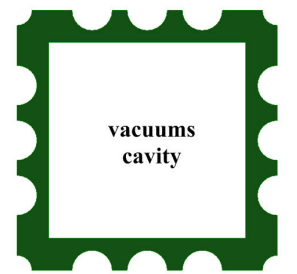

(e)

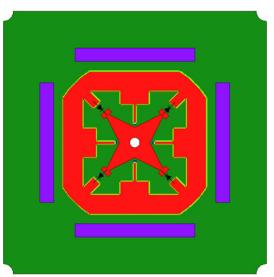

(f)
Fig. 1. Configuration and geometry of the proposed reconfigurable reflectarray unit-cell based on the polarization turning concept. (a) 3D-view, (b) Top-view, (c) Side-view, (d) Impedance-match layer, (e) Coupling layer, and (f) Polarization-turning layer.

PIN diodes are laid symmetrically in two diagonal lines on a square grid to control the induced currents. Polarization of one PIN diode pair in a diagonal line is radial, and the other is contrary.

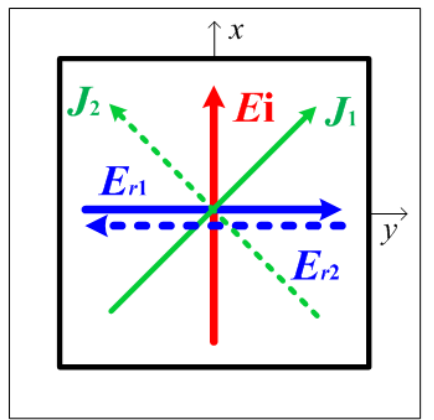

Fig. 2. Diagram of the incident and reflected fields with the induced currents in the unit-cell of the polarization twist.

According to the constitutive relation $\vec{J}=\sigma \cdot \vec{E}$ in Maxwell's equations, the surface current is calculated. When the incident 
field $\vec{E}_{i}=E_{x} \hat{x}$ (or $E_{y} \hat{y}$ ) is impinging upon the unit-cell, the reflected field $\vec{E}_{r}$ can be formed for the states respectively. For state " 1 " of the polarization twist, the current distribution on the patches in the polarization-turning layer is defined as

$$
\vec{J}_{1}=J_{1 x} \hat{x}+J_{1 y} \hat{y}, \vec{J}_{1}=\sigma \cdot\left(\vec{E}_{i}+\vec{E}_{r 1}\right) .
$$

In the same way, for state " 0 ", the current is defined as

$$
\vec{J}_{2}=J_{2 x} \hat{x}+J_{2 y} \hat{y}, \vec{J}_{2}=\sigma \cdot\left(\vec{E}_{i}+\vec{E}_{r 2}\right) .
$$

Due to the symmetrical structure and the special locations of the diodes, currents on the patches in the polarization turning layer in two states flow in the orientations of $\hat{x} \pm \hat{y}$ respectively, as shown in Fig. 2, which comply with the following equations

$$
J_{1 x}=J_{2 x}, J_{1 y}=-J_{2 y}
$$

Adding (1) and (2) side by side, we obtain

$$
\begin{gathered}
\left(J_{1 x}+J_{2 x}\right) \hat{x}+\left(J_{1 y}+J_{2 y}\right) \hat{y}=\sigma \cdot\left(2 E_{x} \hat{x}+\vec{E}_{r 1}+\vec{E}_{r 2}\right) \\
\left(J_{1 x}+J_{2 x}\right) \hat{x}=2 \sigma \cdot E_{x} \hat{x}+\sigma \cdot\left(\vec{E}_{r 1}+\vec{E}_{r 2}\right)
\end{gathered}
$$

It follows from (4) that

$$
\left(\vec{E}_{r 1}+\vec{E}_{r 2}\right) \cdot \hat{y}=0
$$

From the space match condition required for reflectarray, we have

$$
\vec{E}_{r 1} \cdot \hat{x}=0, \vec{E}_{r 2} \cdot \hat{x}=0
$$

It follows from (5) and (6) that $\vec{E}_{r 1}+\vec{E}_{r 2}=0$, i.e.

$$
\vec{E}_{r 1}=-\vec{E}_{r 2}
$$

Eqn. (7) indicates that in states " 1 " and " 0 ", the phase difference of the reflected electric fields is $180^{\circ}$, with equal amplitudes. Hence, this is the theoretical basis for the proposed 1-bit phase-resolution scheme derived from the polarization turning concept.

The inclined orientation of PIN diodes twists polarization of the reflected electric field by $\pm 90^{\circ}$ referring to the incident field. The structural symmetry in the unit-cell offers two directions for the polarization twist, and the switchable states of two diode PIN pairs dictate whether a right-hand or left-hand directional polarization twist is performed.

By using the polarization turning concept, a Ku-band reflectarray unit-cell with 1-bit phase resolution is developed. By employing the periodic boundary condition, it is possible to assess performance of the element coupling within the array. With the plane wave excitation of the normal and oblique incidences, the unit-cell is analyzed using the commercial electromagnetic software Ansys HFSS [7].

Numerical analysis has been carried out using Floquet boundary approach for the unit-cell with size $13 \times 13 \mathrm{~mm}^{2}$. The unit-cell is excited by the plane waves, and their electric fields are perpendicular to the grid of the periodic boundaries in the model. In the pre-installing situation for the reflectarray, the oblique incidence is focused, and the unit-cell performance under $30^{\circ}$ incidence angle needs to be evaluated. Fig. 3 (a) shows the simulated scattering parameter results for the normal and oblique incidences, showing the phase difference between the polarization transfer coefficients $\mathrm{S}_{21}$ in two states. The $180^{\circ}$ step for 1-bit phase-shift resolution is confirmed in Fig. 4. To validate the unit-cell design, experiment in the waveguide simulator (WGS) is established for the $30^{\circ}$ oblique incidence situation, and a proof-of-concept $2 \times 2$ array of the designed

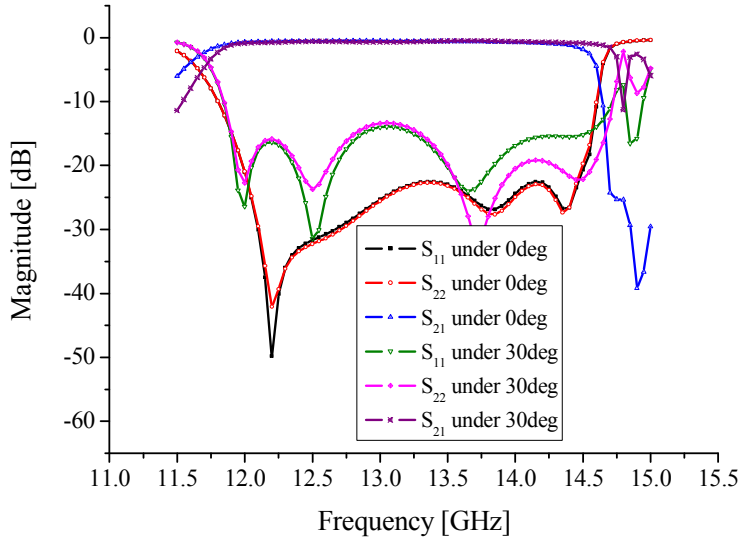

(a)

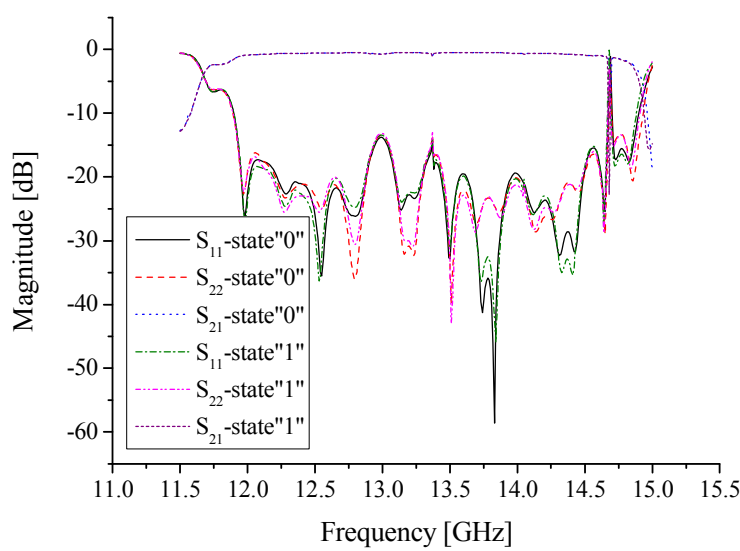

(b)

Fig. 3. Scattering parameters of the proposed reflectarray unit-cell in two bias voltage states. (a) Simulation under Floquet Boundary Condition, and (b) Experiment in the waveguide simulator.

unit-cell is excited in an orthomode transducer (OMT) with aperture size $26 \times 26 \mathrm{~mm}^{2}$. The WGS aperture size depends on the incident angle in the simulated situation for oblique incidence. In order to maintain the integrity of unit-cells within the reflectarray, an $m \times n$ subarray is generally chosen as the sample in the WGS. In our simulation, an array with $2 \times 2$

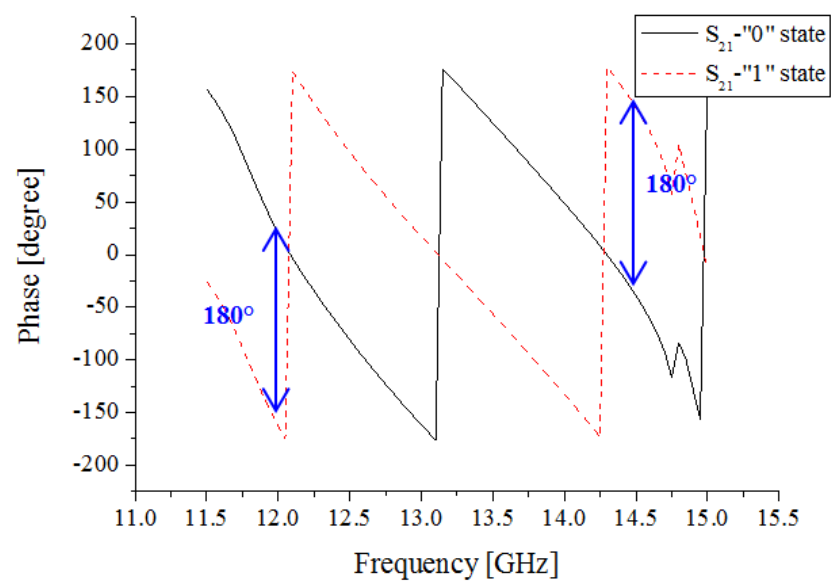

Fig. 4. Simulated phase of the polarization transfer coefficients for 2 states in the proposed unit-cell excited by the incident plane wave with $30^{\circ}$ incidence angle. 
unit-cells is inserted in the OMT for the dual-polarization analysis to confirm the performance with $30^{\circ}$ oblique incidence angle. The reflections in two orthogonal polarizations and their transformation coefficients are presented in Fig. 3 (b). Due to the resonance in the cavity formed by the OMT and the sample, the scattering parameter results shown in Fig. 3(b) do not correlate completely with those from the plane wave excitation in the open air, as shown in Fig. 3(a). High order modes in the waveguide are produced for the overmoded waveguide aperture in the WGS test port for measuring the $2 \times 2$ array.

Additionally, the coaxial structure in the RF-DC isolation circuitry for biasing control increases the isolation level. Simulated results indicate that more than $36 \mathrm{~dB}$ RF decoupling for the oblique incidence is obtained at DC-control point of the bias voltage.

Simulations and experiments using the WGS demonstrate that the unit-cell integrated with lossy PIN diodes can achieve the 1-bit phase-shift for RRAs with low loss $(0.5-1 \mathrm{~dB})$ and low reflections (nearly $-15 \mathrm{~dB}$ ) for both polarizations in an approximate $20 \%$ relative bandwidth, as shown in Fig. 3 . This unit-cell is relatively simple in structure when compared with other solutions, such as the complex FR-4 cavity in [4]. The non-complex circuit layout improves the RF-DC isolation, and allows easy integration with active devices, such as PIN diodes or MEMS components. The novel unit-cell design is validated by the simulations and the scattering performance in the WGS. As is shown, the unit-cell with multilayer structure operates in the $\mathrm{Ku}$ band, and reflects the linearly polarized waves with the polarization turning, which can be switched by controlling the states of PIN diodes.

Compared with the unit-cell using the slot circuit described in [4], the proposed unit-cell utilizes the polarization-turning circuit based on the microstrip line, which is easily derived according the current expression from (1) to (7). The dual balance-located PIN diode pairs are connected parallel in the polarization-turning layer, thus reducing the biasing drive internal resistance. This scheme means the thermal condition for the unit-cell is improved, and the PIN diodes in this design can work more reliably. Simulated results also illustrate that the insensitivity to the incidence angle is also strengthened due to the symmetrical location of four PIN diodes on a cross-shaped microstrip. The circuitry in the polarization- turning layer for RF-DC isolation is also inspired by design of the patch antenna.

\section{OFFSET-FED RECONFIGURABLE REFLECTARRAY ANTENNA CONFIGURATION}

In the RRA design, the element spacing $d$ is set according to the beam-scanning range. Based on the array theory [9], the grating lobe location $\theta_{G L}$ depends on the main beam scanning angle $\theta_{0}$ and the element spacing $d$. The operating frequency band for the RRA design is set as $12.0-14.5 \mathrm{GHz}$, the element spacing is chosen as $13 \mathrm{~mm}$, about $0.63 \lambda_{\text {min }}$, and the beam-scanning range is set to $\pm 30^{\circ}$ mainly on the basis of the antenna application background. It is reckoned that the element spacing meets the criterion for beam-scanning to $\pm 35^{\circ}$ without grating lobes. When the main beam scans to $\pm 40^{\circ}$, though the grating lobes will appear at the location $\theta_{G L}=71.6^{\circ}$, the roll-off of the element scattering pattern attenuates the grating lobe, and the array configuration assigns this RRA with the ability of $\pm 40^{\circ}$ scanning range.

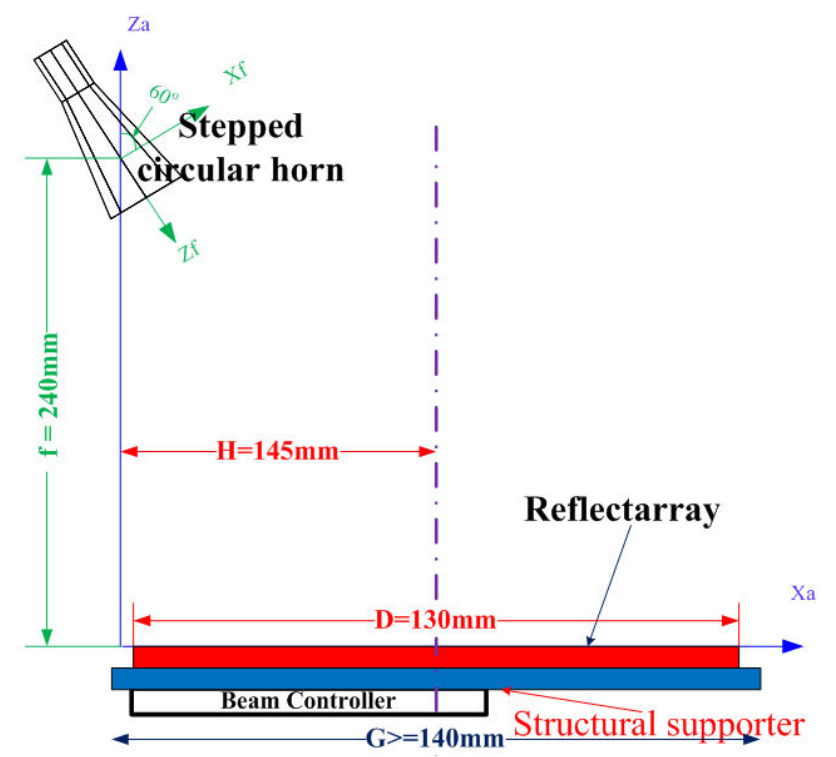

Fig. 5. Configuration and geometry of the offset-fed reconfigurable reflectarray antenna using the stepped circular horn.

By using the novel unit-cell with single-bit phase resolution, a RRA prototype with $10 \times 10$ elements is fabricated and measured to validate its beam- scanning ability. The scale of the RRA prototype should be chosen properly to confirm the design method by performance analysis. It will be a great challenge for conducting a full wave analysis (FWA) if the scale of the reflectarray increases greatly. Meanwhile, the beam with suitable angle resolution should be formed to detect the beam-scanning ability and to distinguish direction of the beam in the course of beam scanning.

To verify performance of the unit-cell with polarization twist, the offset-fed reflectarray antenna configuration for the dual polarization and the circular polarization, as shown in Fig. 5, is adopted. The offset-fed reflectarray antenna configuration [10] is of additional frequency-squint effects for the RRAs using 1-bit digital phase shifters, as discussed in $[3,11]$. Due to the specular reflection characteristic of the reflecting elements [12, 13], the wave is reflected to the opposite angle from the incident angle with low error in a wider bandwidth, and the sources as well as the radiation ranges agree with the specular characteristic of the reflecting elements. The main beam could scan in a wide angular range with low squint error. As one typical option, the offset-fed antenna configuration is frequently used in the RRAs. In Fig. 5, high offset value $(145 \mathrm{~mm})$ is set for the offset-fed RRA to eliminate the scattering from the feed, and the angle of the feed axis is $30^{\circ}$ with respect to the normal direction of the reflectarray plane. According to this feeding method, the specular angular range is focused to investigate the beam- steering performance.

According to the polarization turning concept, when the incident field $\vec{E}_{i}$ impinges upon the unit-cell, the ideal response function $\vec{f}$ for the unit-cell defines the reflected field 
$\vec{E}_{r}=\vec{f}\left(\vec{E}_{i}\right)$. According to the reciprocity principle, neglecting the losses, the responses for the incident fields $E_{0} \hat{y}$ and $E_{0} \hat{x}$ are expressed as

$$
\left.\vec{E}_{r}\right|_{E_{0} \hat{y}}=\vec{f}\left(E_{0} \hat{y}\right)= \pm E_{0} \hat{x},\left.\vec{E}_{r}\right|_{E_{0} \hat{x}}=\vec{f}\left(E_{0} \hat{x}\right)= \pm E_{0} \hat{y}
$$

Here symbol " \pm " indicates two states of the unit-cell with different directions of the polarization twist. When impinged upon by the incident field $\vec{E}_{i}=E_{x} \hat{x}+E_{y} \hat{y}, E_{x} \neq 0, E_{y} \neq 0$, for arbitrary polarization angle in the linearly polarized wave, the reflected field is expressed as

$$
\vec{E}_{r}=\vec{f}\left(\vec{E}_{i}\right)= \pm\left(E_{y} \hat{x}+E_{x} \hat{y}\right)
$$

Clearly, the polarization of the reflected field is not orthogonal to the polarization of the incident field, if the incident field is not parallel to $\hat{x}$ or $\hat{y}$, the borders of the unit-cell. We also have

$$
\left|\vec{E}_{r} \cdot \vec{E}_{i}^{*}\right|=\left| \pm\left(E_{x} E_{y}+E_{y} E_{x}\right)\right|=2 E_{x} E_{y} \neq 0
$$

Eqn. (10) reveals that the polarization twist with perfect $\pm 90^{\circ}$ angle is available for the excited plane waves only when the electric fields are perpendicular to the grid of the array, because PIN diodes are laid in two diagonal lines of the square grid. If the $45^{\circ}$ linear polarization referenced to the square grid of the unit-cell is excited, the polarization of the reflected wave coincides with the incident wave, and no polarization twist occurs.

When the circularly polarized incident field $\vec{E}_{i}=E_{0} \hat{u}=$ $\frac{E_{0}}{\sqrt{2}}(\hat{x} \pm j \hat{y})$ with the wave direction $\hat{k}$ impinges upon the unitcell, for state " 1 " in the unit-cell, the reflected field $(-\hat{k})$ can be obtained by the response function.

$$
\begin{gathered}
\vec{E}_{r 1}=\vec{f}\left(\vec{E}_{i}\right)=\frac{E_{0}}{\sqrt{2}} \vec{f}(\hat{x} \pm j \hat{y})=\frac{E_{0}}{\sqrt{2}}(\vec{f}(\hat{x}) \pm j \vec{f}(\hat{y})) \\
=\frac{E_{0}}{\sqrt{2}}(\hat{y} \pm j \hat{x})= \pm \frac{E_{0}}{\sqrt{2}} j(\hat{x} \mp j \hat{y})= \pm E_{0} j \cdot \hat{u}^{*}
\end{gathered}
$$

While for state " 0 " in the unit-cell, the reflected field $(-\hat{k})$ can be calculated similarly.

$$
\begin{aligned}
& \vec{E}_{r 0}=\frac{E_{0}}{\sqrt{2}}(\vec{f}(\hat{x}) \pm j \vec{f}(\hat{y}))=\frac{E_{0}}{\sqrt{2}}(-\hat{y} \mp j \hat{x}) \\
& =\mp j \frac{E_{0}}{\sqrt{2}}(\hat{x} \mp j \hat{y})=\mp E_{0} j \cdot \hat{u}^{*}
\end{aligned}
$$

The polarizations of the reflected fields are identical with the incident fields in the circularly polarized excitation case for both states " 1 " and " 0 " from the polarization criterion [14] $\left.<\hat{k}, \hat{u}\rangle=<-\hat{k}, \hat{u}^{*}\right\rangle$ defined by the unitary vector. This feature is distinct from the polarization change in the circularly polarized reflector antenna. Thus, it can be deduced that in the offset-fed reflectarray antenna configuration, the polarization of the beam is the same as that of the feed. If the feed is righthand circularly polarized (RHCP), the RHCP beam can be formed by the reflectarray, and vice versa. Eqns. (11) and (12) also reveals that a $180^{\circ}$ reflected field phase difference between two states is still achieved by the proposed unit-cell with single-bit phase shift in the circularly polarized excitation case.

When the proposed dual-polarized unit-cell with single-bit phase resolution is used in the RRAs, the antenna performance is heavily influenced by the feed assembly, hence careful design of the antenna feed is required. The unit-cell can reflect the dual-polarized waves because of its symmetrical configuration, which is combined with the dual-polarized feed in the reflectarray antenna.

In the offset-fed RRA, the stepped circular horn [8] is introduced for realizing the dual polarization, which has a profile of 5-segment polygonal line (output aperture of $60.90 \mathrm{~mm}$, and length of $106.12 \mathrm{~mm}$ ). The stepped circular horn is optimized for the low cross-polarization level and the phase center location $(10 \mathrm{~mm})$ near the radiating aperture with a Gaussian beam of nearly $18 \mathrm{dBi}$ peak gain and less than $-25 \mathrm{~dB}$ reflection coefficients. The stepped circular horn is assumed to be axis symmetric, and its polarization characteristic is defined by the excitation, specifically the feed assembly cascaded to the horn produces the corresponding polarization. For the dual linearly polarized reflectarray, the stepped circular horn is combined with an OMT. If the axis symmetric horn is excited by a circularly polarized source integrated with a circular polarizer, such as a septum polarizer or a corrugated waveguide polarizer, the circular polarization can be produced in the offset-fed reflectarray antenna.

The stepped circular horn works with good performance in $13.0-14.5 \mathrm{GHz}$ band. Certainly this type of horn may be replaced by a corrugated horn that meets the requirements in the total band strictly. Because of its simple structure and easy fabrication, the stepped circular horn is chosen in the offset-fed reflectarray antenna configuration to confirm the unit-cell design and the performance under the circularly polarized condition.

The definition of ports with polarization discrimination can be decided by the feature and criterion in the circularly polarized feed without considering the reflection in the antenna, as interpreted above. The performance of the offset-fed RRA excited by the circularly polarized primary source is given in Section IV, and the circular polarization characteristic interpreted above is confirmed by the results below from the full wave analysis.

\section{Performance AnAlysis And Measurements}

A full wave analysis was performed to validate the design and assess the performance of RRAs. This section presents the performance analysis of the offset-fed RRA. In the RRA, each unit-cell is controlled independently, and the array synthesis is implemented for the single-bit phase shifts to form the specified direction after phase quantization and pattern confirmation by applying Fourier transform to get the far field pattern from the aperture field. Consequently, for each beam with different direction certain distribution consisting of the controlled logically independent unit-cell states in the array can be computed, and the corresponding control code matrix is defined. Each beam has its definitive control code matrix, and one control code matrix in the obtained data base for beamscanning forms the beam different from others.

For the dual-polarized offset-fed RRA shown in Fig. 5, its performance can be analyzed for different polarization excitations. Arbitrary beam direction (e.g. $\theta=40^{\circ}, \varphi=60^{\circ}$ ) is set up, and the control code matrix is calculated for the beam forming at $13.5 \mathrm{GHz}$. The excited field in the reflectarray aperture is calculated from the horn field and antenna configuration. The phase distribution in the aperture is 
determined by the beam direction. The required phase shifts in the array are discretized by $180^{\circ}$, and the states composed of " 1 " and " 0 " (corresponding to bias voltages " $+5 \mathrm{~V}$ " and " $-5 \mathrm{~V}$ ") in the array define the control code matrix, as shown in Table I for the beam at $13.5 \mathrm{GHz}$ with the expected direction. The influence of the phase quantification has been confirmed by transforming the phase-discretized field in the aperture to get the far field pattern by fast Fourier transform (FFT) algorithm, as shown in Fig. 6.

TABLE I

REFLECTARray CONTROL CODE MATRIX OF THE BEAM IN THE EXPECTED DIRECTION AT 13.5GHZ FOR THE OFFSET-FED CONFIGURATION

\begin{tabular}{|c|c|c|c|c|c|c|c|c|c|c|}
\hline Row Col. & 1 & 2 & 3 & 4 & 5 & 6 & 7 & 8 & 9 & 10 \\
\hline 1 & 1 & 1 & 1 & 1 & 1 & 1 & 0 & 0 & 0 & 1 \\
\hline 2 & 0 & 0 & 0 & 0 & 0 & 0 & 1 & 1 & 1 & 0 \\
\hline 3 & 1 & 1 & 1 & 1 & 1 & 0 & 0 & 0 & 0 & 1 \\
\hline 4 & 0 & 0 & 0 & 0 & 1 & 1 & 1 & 1 & 0 & 0 \\
\hline 5 & 1 & 1 & 1 & 0 & 0 & 0 & 0 & 1 & 1 & 1 \\
\hline 6 & 1 & 1 & 1 & 1 & 1 & 1 & 0 & 0 & 0 & 1 \\
\hline 7 & 0 & 0 & 0 & 0 & 0 & 1 & 1 & 1 & 0 & 0 \\
\hline 8 & 1 & 1 & 0 & 0 & 0 & 0 & 0 & 1 & 1 & 1 \\
\hline 9 & 1 & 1 & 1 & 1 & 1 & 0 & 0 & 0 & 0 & 1 \\
\hline 10 & 0 & 0 & 1 & 1 & 1 & 1 & 1 & 0 & 0 & 0 \\
\hline
\end{tabular}

The offset-fed RRA was analyzed using HFSS according to the control code matrix given in Table I. The results, presented in Fig. 7 and Table II, correspond to a beam in the expected direction $\left(\theta=40^{\circ}, \varphi=60^{\circ}\right)$. It can be discovered that the polarization of the antenna beam is different from that of the feed when the feed is linearly polarized, parallel to the feed coordinate axis Xf or Yf. However, for the circularly polarized feed excitation, the reflection from the reflectarray with polarization twist does not change the polarization, and the beam keeps the same polarization to the primary source. The performance is stable in the operating frequency band, and the variation of antenna peak gain is limited to be less than $3 \mathrm{~dB}$, as shown in Table II. The peak efficiency of this antenna, which appears to be less than $15 \%$, is mainly limited by some factors as follows. (1) RF loss in the element (about $1 \mathrm{~dB}$ absorbed by the resistance in PIN diodes), (2) Phase error caused by two phase states ( 0 degree and 180 degree), about $5.4 \mathrm{~dB}$ reduction in directivity as shown in [17], (3) Space-feeding efficiency

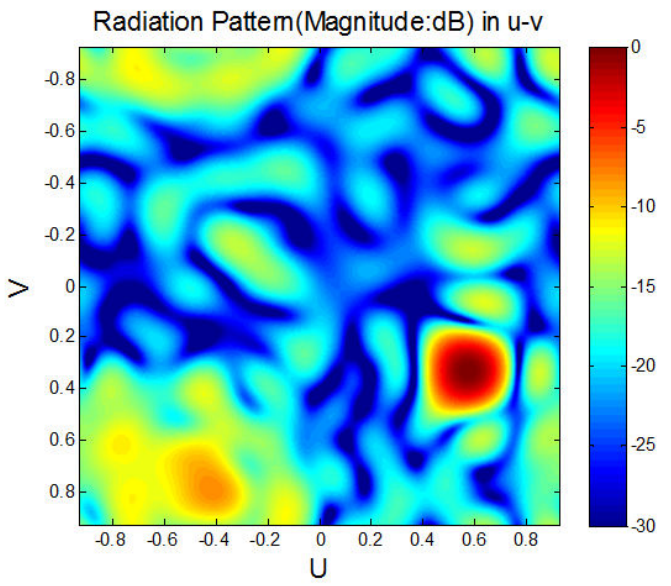

Fig. 6. Calculated radiation pattern of the beam direction $\left(\theta=40^{\circ}, \varphi=60^{\circ}\right)$ in the offset-fed reconfigurable reflectarray antenna at $13.5 \mathrm{GHz}$ after FFT. (about 70\%) in the offset-fed configuration, and (4) Some other losses due to the reflection and scattering. The RRA has the beam-scanning ability with easy control at the expense of the efficiency. It can be also noticed from Table II that the antenna gain of the vertical polarization at $14.5 \mathrm{GHz}$ in the case of horizontally polarized feed excitation drops by about $1.6 \mathrm{~dB}$, compared with its counterpart. The reason is that the incident angles for some unit-cells in the reflectarray are beyond $30^{\circ}$. This effect can also be supported by the obvious disparity of the reflection coefficients between two polarizations in Fig. 3 (a) at the frequencies near $14.5 \mathrm{GHz}$ in the oblique incidence cases. Some unit-cells in the margin of reflectarray deteriorates the performance for the parallel polarization incidence, and the relation of the electric field to the incidence plane is of importance to the transformation coefficients of polarization in the situations with large incident angles, while the performance maintains well for the vertical polarization incidence.

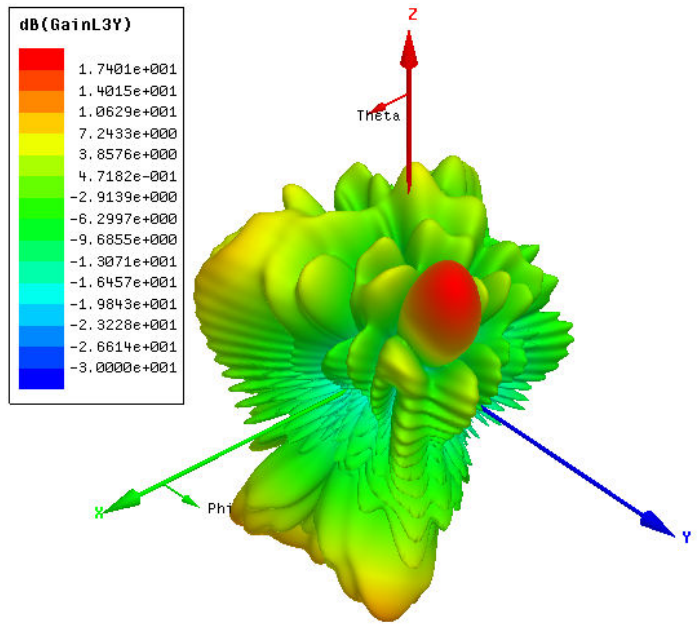

Fig. 7. Simulated radiation pattern of the beam direction $\left(\theta=40^{\circ}, \varphi=60^{\circ}\right)$ in the offset-fed reconfigurable reflectarray antenna at $13.5 \mathrm{GHz}$ after the FWA.

The reflection coefficients of the antenna are still below $-25 \mathrm{~dB}$ in virtue of the offset-fed configuration, and the side lobe levels (SLL) are about -10dB due to the extremely large phase quantification, but the beam in the expected direction is formed in the operating frequency band, and the cross-polarization level in the main beam is approximately $-20 \mathrm{~dB}$. The detailed results are displayed in Fig. 8.

TABLE II

PERFormance SumMary OF THE OFFSET-Fed RECONFIGURABLE REFLECTARRAY WITH MULTI POLARIZATION

\begin{tabular}{|c|c|c|c|c|c|}
\hline \multirow{2}{*}{$\begin{array}{c}\text { Polarization } \\
\text { of feed }\end{array}$} & \multirow{2}{*}{$\begin{array}{c}\text { Polarization } \\
\text { of beam }\end{array}$} & \multicolumn{4}{|c|}{ Peak Gain (dBi) } \\
\hline & & $13.0 \mathrm{GHz}$ & $13.5 \mathrm{GHz}$ & $14.0 \mathrm{GHz}$ & $14.5 \mathrm{GHz}$ \\
\hline Horizontal & Vertical & 16.5 & 17.4 & 17.5 & 15.9 \\
\hline Vertical & Horizontal & 16.8 & 17.3 & 17.3 & 17.6 \\
\hline LHCP & LHCP & 16.8 & 17.3 & 17.5 & 16.8 \\
\hline RHCP & RHCP & 16.6 & 17.3 & 17.3 & 16.7 \\
\hline
\end{tabular}

The randomness in choosing the direction for the reconfigurable beam and the obtained antenna performance verify the beam-steering ability of the offset-fed RRA with $180^{\circ}$ phase resolution and the proposed unit-cell based on the 
polarization twist. Deduced from (11) and (12), the relation between the polarization of the antenna beam and the primary source, for the antenna under the circularly polarized excitation, is confirmed by the FWA definitely, after the calculated results are summarized.

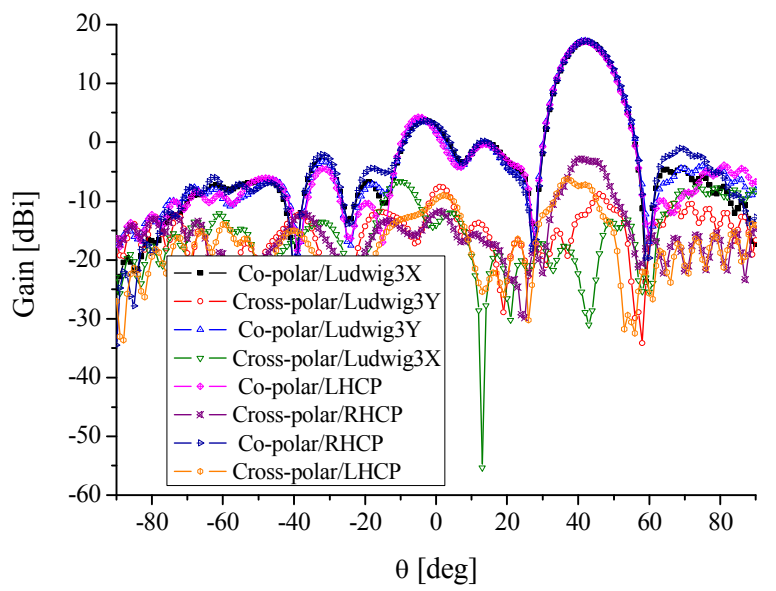

Fig. 8. Comparison of the co-polar and cross-polar radiation patterns (beam direction: $\left.\theta=40^{\circ}\right)$ in $\varphi=60^{\circ}$ cutting plane for the offset-fed reconfigurable reflectarray antenna at $13.5 \mathrm{GHz}$ after FWA.

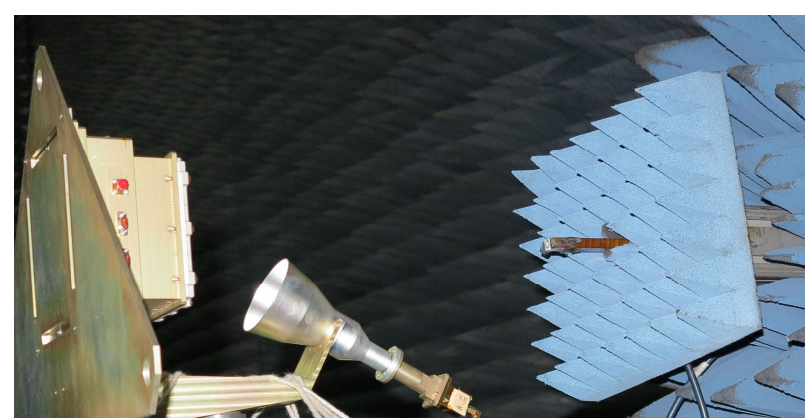

Fig. 9. Prototype of the offset-fed reconfigurable reflectarray antenna under test in the chamber.

A prototype of the offset-fed RRA was manufactured, as demonstrated in Fig. 9, and the antenna prototype was tested by the near field measurement system to obtain the beam-scanning patterns at $13.5 \mathrm{GHz}$ with gains ranged from 15.7 to $16.5 \mathrm{dBi}$.

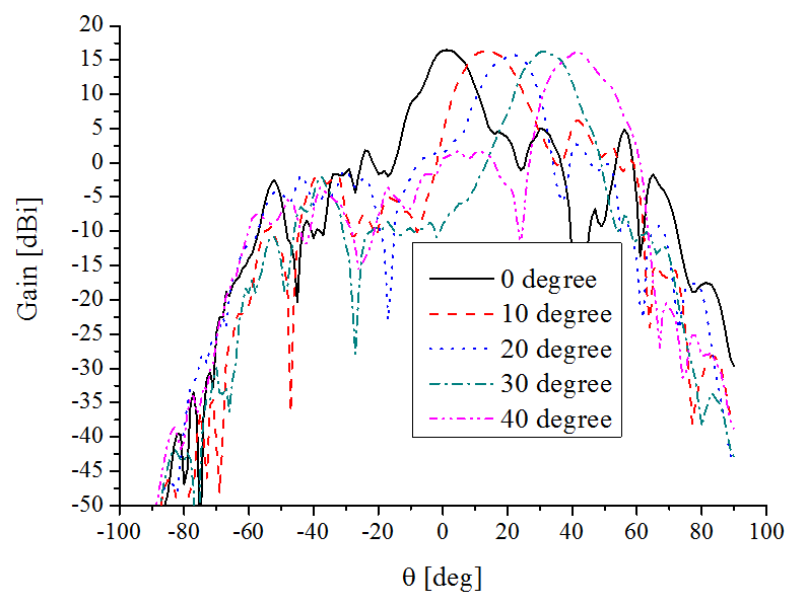

Fig. 10. Measured pattern results for the prototype of the offset-fed RRA to demonstrate the beam-scanning at $13.5 \mathrm{GHz}$.
Measured pattern results are given in Fig. 10 to show that the beam of the single-bit phase-shift RRA can be electronically controlled to scan with a wide range.

In this experiment, the method for computing the control code matrices according to the beam directions is examined, and it works well to predict the antenna beam and to estimate the influence of the phase shift with single-bit resolution. Through the performance analysis and measurements, the novel RRAs with the single-bit phase resolution for Ku-band satellite applications are validated. The polarization characteristics of the offset-fed RRA are analyzed, and its beam- scanning ability is confirmed by the fabricated prototype. Some influences not involved in the array synthesis are assessed in the calculation and experiment.

\section{CONCLUSIONS}

A novel Ku-band multilayer reflectarray with $10 \times 10$ unit cells has been presented and investigated. The dual-polarized unit-cell with $180^{\circ}$ phase resolution, combined with the ability to twist the polarization in the linearly polarized wave, enables the reflectarray to perform beam scanning. The polarization twist is controlled by switching states of the integrated PIN diodes in each unit-cell, thus a control code matrix is required for the complete reflectarray, which defines the direction of the reflected beam. In addition, the unit-cell is able to form a circularly polarized beam, if the primary source is also circularly polarized. This feature is confirmed by the offset-fed RRA, and no polarization change occurred after the reflection of the reflectarray in the circularly polarized excitation case. Also, in the offset-fed RRA the beam in arbitrary direction is designed by this method and analyzed to confirm the beamscanning. The summarized results demonstrate the antenna beam-scanning ability, which are derived from full wave analyses.

In this paper, the design method is outlined for RRAs, including the beam forming and control. Additionally, the designs of associated components for the proposed RRAs are presented, and the principles of the unit-cell and the polarization twist are also discussed. More details and manufacture factors are taken into account to anticipate accurately the final available RRA performance. In spite of low efficiency, compared with conventional phased array antennas, the RRAs based on the single-bit phase shift remain of interest due to its low cost and easy realization for the beam control. In future applications, the performance could be enhanced further, and the aperture size will be increased for higher resolution. In this case, the technical advantages of the RRAs with single-bit phase shift will be highlighted.

The polarization twist, utilized in the proposed RRAs, offers frequency independence for wideband performance. This method also has potential for integrating the unit-cell with a polarizing grid in folded reflectarrays, where low structural profiles may be achieved, an important factor for antenna designers.

In conclusion, the numerical study of the RRA, the experimental results from the unit-cell in the WGS and the measurements for the antenna prototype presented here, 
demonstrate that the proposed RRA can be utilized in the Ku-band satellite applications. The proposed RRA with its structural features can also be extended to design antenna products mounted on the suitable position of satellite. The potential for space communications and radar detections [17] urges us to improve the performance of RRAs and to promote the research phase for product qualification in this field. RRAs with larger apertures are on our road map of research, which we aim to present in the near future.

\section{ACKNOWLEDGMENT}

This research is funded by China Scholarship Council and Xi'an Institute of Space Radio Technology (XISRT). The authors would like to thanks the staffs from China Scholarship Council, XISRT and the University of Kent for the support and help. Also Mr. Xudong Wang and Mrs. Xin Liu from XISRT are acknowledged for designing the circumstantial structure and manufacturing the antenna prototype.

\section{REFERENCES}

[1] S. V. Hum and J.Perruisseau-Carrier, "Reconfigurable reflectarrays and array lenses for dynamic antenna beam control: A review," IEEE Trans. Antennas Propag., vol. 62, no.1, pp. 183-198, Jan. 2014.

2] R. Sorrentino, R.V. Gatti, and L. Marcaccioli, "Recent advances on millimetre wave reconfigurable reflectarrays," in Proc. 2009 Europ. Conf. Antennas Propag. (EuCAP 2009), 2009, pp. 2527-2531.

[3] S. Ebadi, R.V. Gatti, and R. Sorrentino, "Linear reflectarray antenna design using 1-bit digital phase shifters," in Proc. 2009 Europ. Conf. Antennas Propag. (EuCAP 2009), 2009, pp. 3729-3732.

[4] S. Montori, F. Cacciamani, R.V. Gatti, E. Carrasco, M. Barba, J. Encinar, and R. Sorrentino, "Wideband dual-polarization reconfigurable elementary cell for electronic steerable reflectarray at Ku-band," in Proc. 2010 Europ. Conf. Antennas Propag. (EuCAP 2010), 2010, pp.1-5.

[5] S. Montori, F. Cacciamani, C. Tomassoni, L. Marcaccioli, and R.V. Gatti, "Novel 1-bit elementary cell for reconfigurable reflectarray antennas," in 2011 Europ. Microwave Conf. (EuMC 2011), 2011, pp. 1288-1291.

[6] H. Kamoda, T. Iwasaki, J. Tsumochi, T. Kuki, and O. Hashimoto, "60-GHz electronically reconfigurable large reflectarray using single-bit phase shifters," IEEE Trans. Antennas Propag., vol. 59, no.7, pp. 2524-2531, July 2011.

[7] HFSS, Ansoft Product Suite. ver. 15, ANSYS, Inc., Canonsburg, PA, USA [Online]. Available: http://www.ansoft.com

[8] K. K. Chan and K.S. Rao, "Design of high efficiency circular horn feeds for multibeam reflector applications," IEEE Trans. Antennas Propag., vol. 56, no.1, pp. 253-258, Jan. 2008.

[9] Arik D. Brown, Electronically scanned arrays MATLAB ${ }^{\circledR}$ Modeling and simulation. CRC Press-Taylor \& Francis Group, 2012.

[10] A. Yu, F. Yang, A. Z. Elsherbeni, J. Huang, and Y. Kim, "An offset-fed $\mathrm{X}$-band reflectarray antenna using a modified element rotation technique," IEEE Trans. Antennas Propag., vol. 60, no.3, pp. 1619-1624, March 2012.

[11] E. Almajali, D.A. McNamara, J. Shaker, and M.R. Chaharmir, "On beam squint in offset-fed reflectarrays," IEEE Antennas Wireless Propag. Lett., vol. 11, pp. 937-940, Aug. 2012.

[12] J. Budhu and Y. Rahmat-Samii, "Understanding the appearance of specular reflection in offset fed reflectarray antennas," in IEEE Int. AP-S Symp. Dig., 2011, pp. 97-100.

[13] R. El Hani and J.-J. Laurin, "Specular reflection analysis for off-specular reflectarray antennas," IEEE Trans. Antennas Propag., vol. 61, no. 7, pp.3575-3581, July 2013.

[14] Y.T. Lo and S.W. Lee, Antenna Handbook, Springer ,1994.

[15] I.Y. Tarn, Y.S. Wang, and S.J. Chung, "A dual-mode millimeter-wave folded microstrip reflectarray antenna," IEEE Trans. Antennas Propag., vol. 56, no.6, pp.1510-1517, June 2008.

[16] S. Montori, R.V. Gatti, R. Sorrentino, S. Dieter, and W. Menzel, "Reconfigurable and dual-polarization folded reflectarray antenna," in 2012 Europ. Microwave Conf., (EuMC 2012), 2012, pp. 735-738.
[17] H. El Gannudi, R.V. Gatti, C. Tomassoni, and R. Sorrentino, "Preliminary design of foldable reconfigurable reflectarray for Ku-band satellite communication," in Proc. 2010 Europ. Conf. Antennas Propag. (EuCAP 2010), 2010, pp. 1-5.

[18] T. Debogovic, S. Susac, and J. Perruisseau-Carrier, "A simple reflectarray cell with 1-bit phase control and polarization flexibility," in Proc. 2013 Europ. Conf. Antennas Propag. (EuCAP2013), 2013, pp. 2717-2720. 